Pacific

Journal of

Mathematics

A REMARK ON DEGENERATE SINGULARITIES

IN THREE DIMENSIONAL RICCI FLOW

YU DING 


\title{
A REMARK ON DEGENERATE SINGULARITIES IN THREE DIMENSIONAL RICCI FLOW
}

\author{
YU DING
}

\begin{abstract}
We show that a rescaling limit at any degenerate singularity of Ricci flow in dimension 3 is a steady gradient soliton. In particular, we give a geometric description of type I and type II singularities.
\end{abstract}

The study of degenerate singularities of the Ricci flow

$$
\frac{d}{d t} g(t)=-2 \operatorname{Ric} g(t)
$$

was introduced in [Hamilton 1995], where Hamilton first described nondegenerate neck-pinching. Roughly speaking, one starts the Ricci flow on a dumbbell-shaped 3 -manifold, with the neck diffeomorphic to $S^{2} \times[-1,1]$. It is expected that the neck shrinks in the $S^{2}$ direction, where the curvature is very positive, and, at the same time, stays relatively stationary in the $\mathbb{R}$ direction, where the curvature is slightly negative. After some time, the neck pinches off and forms a singularity. One step further, Hamilton purposed the notion of degenerate neck-pinching: reduce the left half of the dumbbell into a critical size and then start the Ricci flow. It is expected that after some time, all of the left half of the dumbbell pinches off, and forms a singularity like a horn growing out of the (remaining) right half of the dumbbell. See [Hamilton 1995] for further descriptions and some very inspiring pictures.

Recall that a solution $X$ develops a type I singularity if $X$ goes singular at time $T$ and the scalar curvature $R(x, t)$ satisfies

$$
\limsup _{t \rightarrow T^{-}}(T-t) \cdot \sup _{x \in X} R(x, t)<\infty .
$$

If this lim sup is $\infty$, we say the singularity is of type II.

In this paper, we prove that in dimension 3, there is no noncollapsing, noncompact ancient solution of positive curvature that develops a type I singularity; see Theorem 2.4. Conjecturally, such ancient solutions cannot develop type II singularities either. So this can be viewed as a first step towards Perelman's conjecture that the Bryant soliton, which does not develop a singularity at all, is the only

MSC2000: 53C44.

Keywords: Ricci flow, singularity. 
noncollapsing, noncompact, ancient solution of positive curvature; see [Perelman 2002, lines 2-9 on page 32].

As a corollary, we prove that a rescaling limit of a degenerate singularity of Ricci flow is a steady gradient soliton; see Theorem 3.23. The precise definition of a degenerate singularity, given in Definition 1.7, is based on Perelman's notion of canonical neighborhoods. In the most interesting cases, the shape of a singularity is either a cylinder, that is $S^{2} \times \mathbb{R}$, or a cap, which looks like a 3-dimensional paraboloid of revolution. Roughly speaking, a degenerate singularity happens if there is a sequence of points $\left(p_{i}, t_{i}\right)$ with $t_{i} \rightarrow T^{-}$and $R\left(p_{i}, t_{i}\right) \rightarrow \infty$, and a neighborhood of $\left(p_{i}, t_{i}\right)$ is cap-like. Our definition is a geometric one that reflects Hamilton's original picture [1995]. On the other hand, as we will see later in this paper, this geometric definition is equivalent to the singularity being of type II.

We start by reviewing some of Perelman's results [2002; 2003]; for more details, see [Cao and Zhu 2006; Kleiner and Lott 2006; Morgan and Tian 2007]. In Section 2 we estimate Perelman's $l$ functional and the reduced volume $\widetilde{V}$ on ancient solutions, and prove Theorem 2.4. It follows that a rescaling limit of a degenerate singularity is either an eternal solution or an ancient solution that develops a type II singularity. In both cases, we need to take a further rescaling limit in forward time; we treat certain issues related to this in Section 3. We also show that under the assumption of degenerate singularity, at least one cap-like region will become singular at time $T$ : it is not possible that all cap regions remain nonsingular at $T$. Finally we use a theorem of Hamilton [1993a] to conclude that the final rescaling limit is a steady soliton. Our arguments are similar to Perelman's compactness/convergence methods that were used extensively in his papers [2002; 2003].

For previous works on neck-pinching, see [Angenent and Knopf 2004; 2007] and the books [Chow et al. 2006; 2007]. In [Chow and Knopf 2004] there is a detailed treatment of nondegenerate neck-pinching in Chapter 2, and a discussion of degenerate neck pinching in pages 62-66. Very recently Gu and Zhu [2007] constructed an example of type II singularity in the radial symmetric case. See also [Garfinkle and Isenberg 2008].

\section{Notation and definitions}

All manifolds we consider in this paper are of dimension 3 . We write $R(x, t)$ for the scalar curvature at $(x, t)$; Rm is the full curvature tensor. The Hamilton-Ivey pinching inequality [Hamilton 1999] says that, if in the beginning the curvature is bounded from below by -1 , we have

$$
\mathrm{Rm} \geq-\phi(|R|),
$$


where $\phi$ is a nonnegative function such that $\lim _{r \rightarrow \infty} \phi(r) / r=0$. In particular, when $R$ is large, the full curvature $\mathrm{Rm}$ is dominated by the scalar curvature $R$.

We follow some notation of Perelman [2002; 2003]. Assume $g(t)$ is a family of metrics on a manifold $M$ that evolve under the Ricci flow. $B(x, t, r)$ denotes the metric ball centered at $x$ of radius $r$ with respect to the metric $g(t)$. One defines the parabolic neighborhood

$$
P(x, t, r,-\Delta t)=B(x, t, r) \times[t-\Delta t, t] .
$$

When we say two sets $\left(U_{1}, p_{1}, t_{1}\right)$ and $\left(U_{2}, p_{2}, t_{2}\right)$ are $\varepsilon$-close, we first rescale $U_{1}$ and $U_{2}$ so that $R\left(p_{1}, t_{1}\right)=R\left(p_{2}, t_{2}\right)=1$. Then $\varepsilon$-close means these rescaled sets are $\varepsilon$-close in the $C^{6}$ topology. There is a similar notion of $\varepsilon$-closeness between parabolic neighborhoods.

Since we deal only with finite time singularities, all solutions are noncollapsing, by [Perelman 2002, 7.3]. In particular, if a sequence of parabolic neighborhoods admits a uniform curvature bound, we can take a pointed limit over a subsequence.

An ancient solution is a solution that exists on the time interval $(-\infty, T)$ for some $T \in \mathbb{R}$. The cylinder $S^{2} \times \mathbb{R}$, with the $S^{2}$ direction evolving under the Ricci flow, is an important example. A set $(Z, z)$ evolving under the Ricci flow over time $[-t, 0]$ is called a strong $\varepsilon$-neck if, after rescaling the metrics by $R(z, 0)$, $Z$ is $\varepsilon$-close to an evolving cylinder of length $\varepsilon^{-1}$ from time $-\varepsilon^{-1}$ to 0 .

Definition 1.2. The caliber of a cylinder $Y=S^{2} \times \mathbb{R}$ is $R^{-1}$, where $R$ is the scalar curvature of $Y$.

Clearly the caliber of a cylinder $Y$ is just half of the square of its radius. The caliber equals to the time the cylinder takes to go singular (that is, to shrink into the real line $\mathbb{R}$ ).

Proposition 1.3 (Perelman). Let $\varepsilon>0$. Assume $M$ is compact, and the initial metric $g(0)$ satisfies the curvature bound $|\operatorname{Rm} g(0)| \leq 1$, and, for all $p \in M$, $\operatorname{Vol}_{g(0)} B_{1}(p) \geq 10^{-1}$.

Then there exists $r_{0}>0$ such that, whenever $R\left(x_{1}, t_{1}\right)>r_{0}^{-2}$, the neighborhood $P\left(x_{1}, t_{1}, R(x, t)^{1 / 2} \varepsilon^{-1},-\varepsilon^{-1} R(x, t)\right)$ under the rescaled metric $R\left(x_{1}, t_{1}\right) g(t)$ is $\varepsilon$-close to a parabolic neighborhood in an ancient solution; precisely, one of the following:

i) a space form with positive curvature evolving under the Ricci flow,

ii) the cylinder $S^{2} \times \mathbb{R}\left(\right.$ or $\left.S^{2} \times \mathbb{R} / \mathbb{Z}_{2}\right)$ evolving under the Ricci flow,

iii) a compact ancient solution with strictly positive, nonconstant (at each time slice) curvature that is diffeomorphic to $S^{3}$ or $\mathbb{R}^{3}$,

iv) a noncompact ancient solution to the Ricci flow with strictly positive curvature. 
Moreover, on $P\left(x_{1}, t_{1}, R(x, t)^{1 / 2} \varepsilon^{-1},-\varepsilon^{-1} R(x, t)\right)$ there is a uniform gradient estimate

$$
|\nabla R(x, t)| \leq C_{1} R^{3 / 2}, \quad\left|\partial_{t} R\right|(x, t) \leq C_{2} R^{2} .
$$

For a proof, see Theorem 12.1 of [Perelman 2002], together with Section 1 of [Perelman 2003]. The possibilities above give a rough classification of noncollapsing ancient solutions of nonnegative curvature in dimension 3. The parabolic neighborhood $P$ is called a canonical neighborhood.

Both types of ancient solutions - (iii), in sufficiently ancient time, and (iv), in all time - contain a piece of an evolving cylinder. See [Perelman 2002, Sections 11 and 12] and especially [Perelman 2003, 1.4].

The following result allows us to make a convention so that options (i)-(iv) appear in a mutually exclusive way:

Proposition 1.5 (Perelman). Let $X$ be an ancient, noncollapsing 3-dimensional ancient solution with bounded nonnegative curvature, nonconstant at each time slice. Then, at each time ( say $t=0), X$ can be decomposed into a "cap part" $X_{C}$ and a "tube part" $X_{T}$, either of which may be empty. $X_{T}$ is connected. $X_{C}$ contains no $\varepsilon$-neck as a subset, has at most two connect components and is connected when $M$ is noncompact. The boundary components (if any) of $X_{C}$ and $X_{T}$ are diffeomorphic to $S^{2}$.

Each connected component of $X_{C}$ is compact. For all $p, q$ in the same connected component of $X_{C}$, we have

$$
R(p, 0) \leq A(\varepsilon) R(q, 0), \quad \operatorname{Diam} X_{C} \leq D(\varepsilon) R(p, 0)^{-1 / 2} .
$$

Every point in the second part, $X_{T}$, is the center of a strong $\varepsilon$-neck.

When $X_{C}$ is connected, it is diffeomorphic to either $S^{3}, R P^{3}$, or the ball $D^{3}$. When $X_{C}$ is not connected and therefore has two components, one component of $X_{C}$ is diffeomorphic to $D^{3}$ and the other is diffeomorphic to either $D^{3}$ or $R P^{3}-D^{3}$.

Most of this is proved in Section 11, especially 11.8, of [Perelman 2002]. The last part concerning the topology of $X_{C}$ can be found in Section 1 of [Perelman 2003]; See also [Morgan and Tian 2007, Chapter 9; Kleiner and Lott 2006; Cao and Zhu 2006]. We see that if $X$ is noncompact, then either $X=S^{2} \times \mathbb{R}$, or $X$ is an approximate tube being connected, through one $S^{2}$ boundary, to a cap that is diffeomorphic to $D^{3}$ or $R P^{3}-D^{3}$. In the later case $X$ is isometric to $S^{2} \times \mathbb{R} / \mathbb{Z}_{2}$.

We make the convention that, if the canonical neighborhood is close to the $X_{T}$ part of a noncompact ancient solution, we classify it into case (ii), instead of (iv), of Proposition 1.3. Therefore, if we classify a canonical neighborhood to be case (iv), the neighborhood contains (and is quasi-isometric to) $X_{C}$. We will not consider cases (i) and (iii) of Proposition 1.3, because if we have to classify a canonical 


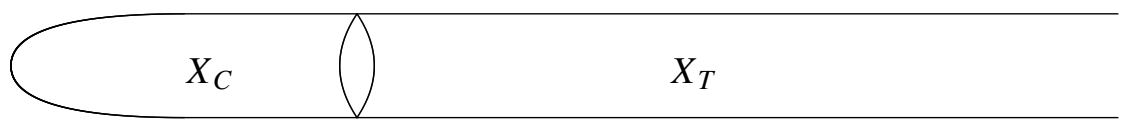

Figure 1. Case (iv) in Proposition 1.3.

neighborhood into these two cases, by taking further compactness arguments, we see the solution will have positive curvature after a very short time. Then the whole solution will become singular and at the same time get rounder and rounder by Hamilton's theorem [1982]; these are all of type I and should not be classified as a degenerate singularities.

With this information, we give a definition of a degenerate singularity of Ricci flow in dimension 3:

Definition 1.7. Assume $M$ is compact and $g(t)$ is a solution to the Ricci flow as in Proposition 1.3 that exists on the time interval $[0, T)$ with $T<\infty$. Assume there is a sequence of points $\left(x_{i}, t_{i}\right)$ so that $\lim _{i \rightarrow \infty} t_{i}=T$ and $\lim _{i \rightarrow \infty} R\left(x_{i}, t_{i}\right)=\infty$; moreover the canonical neighborhood of $\left(x_{i}, t_{i}\right)$ is of case (iv) in Proposition 1.3. Then we say a degenerate singularity happens at time $T$.

A glance at the picture in [Hamilton 1995] suggests that one might also include case (ii) in Proposition 1.3, when the canonical neighborhood is $S^{2} \times \mathbb{R} / \mathbb{Z}_{2}$; we will discuss this possibility in the end of this paper.

It is possible that the solution goes singular everywhere, that is, the scalar curvature goes to infinity everywhere as $t \rightarrow T^{-}$. Perelman's standard solution [2003, Section 2] is a noncompact example of this. At this moment, we don't know a compact example that becomes extinct everywhere while developing a degenerate singularity. If a compact solution becomes extinct everywhere at $T$, the topology of $M$ is quite simple; see [Perelman 2003, Sections 3 and 4].

Proposition 1.8 (Perelman). Given any $\varepsilon$, there exists a small constant $\eta>0$ such that the following is true:

Assume $X$ is a noncollapsing, noncompact, ancient solution of nonnegative, bounded curvature defined for time $t \in(-\infty, 0]$. Assume $\gamma$ is a minimal geodesic segment at time 0 , with two end points $p_{1}$ and $p_{2}$, and $p$ is a point on $\gamma$. Assume

$$
R\left(p_{1}, 0\right)>\eta^{-1}, \quad R(p, 0)=1, \quad R\left(p_{2}, 0\right)<\eta .
$$

Then $d\left(p_{1}, p\right)>\varepsilon^{-1}, d\left(p, p_{2}\right)>\varepsilon^{-1}$, and $B\left(p, 0, \varepsilon^{-1}\right)$ is $\varepsilon$-close to a subset in a cylinder of caliber 1 .

This follows from Perelman's compactness theorem [Perelman 2002, 11.7]; roughly, if this is not true, take a limit of counterexamples. By Perelman's curvature bound [Perelman 2002, last three lines in page 30], we see the distance from $p$ to 
$p_{1}, p_{2}$ goes to $\infty$. Therefore the limit contains a line and splits; it must be the cylinder and that is a contradiction. See [Cao and Zhu 2006; Kleiner and Lott 2006; Morgan and Tian 2007] for details.

Finally, with nonnegative curvature, a singularity must happen everywhere:

Proposition 1.10 (Perelman). Assume $X$ is a noncollapsing ancient solution of strictly positive curvature. If $X$ develops a singularity at time $T$, then for all $x \in X$,

$$
\lim _{t \rightarrow T^{-}} R(x, t)=\infty .
$$

See Claim 2 in the proof of Theorem 12.1 in [Perelman 2002].

\section{A theorem on ancient solutions}

Definition 2.1. Assume $X$ is a noncollapsing, noncompact ancient solution of strictly positive, bounded curvature, defined for time $(-\infty, 0]$. The caliber of $X$ at time 0 is

$$
\lim _{x \rightarrow \infty} \frac{1}{R(x, 0)} .
$$

This limit exists; in fact, the solution is close to a cylinder with the above caliber when $x \rightarrow \infty$. See Proposition 1.5 and the volume comparison argument in Lemma 2.5.

Since $(X, g(0))$ has bounded curvature, by Shi's theorem [1989a] we can extend the solution for a short time beyond $t=0$. Moreover, over each closed time interval on which the solution exists, the curvature is bounded and positive; see [Shi 1989b, Theorem 4.14]. See also [Morgan and Tian 2007, 12.1] for an alternative argument.

We can give a partial order to the set of all Ricci flow solutions with initial data $(X, g(0))$ having bounded curvature over each closed time interval in which the solution exists: $g_{1} \leq g_{2}$ if $g_{2}$ is an extension (in time) of $g_{1}$. Extensions may or may not be unique. We now consider maximal solutions according to this order:

Lemma 2.3. Assume $g(t)$ is a maximal solution with initial data $(X, g(0))$ having bounded curvature in each time slice. Then $g(t)$ exists on $[0, C)$, where $C$ is the caliber of $(X, g(0))$. If $C<\infty$, then $\lim _{t \rightarrow C^{-}} R(x, t)=\infty$ for all $x \in X$.

Proof. The proof is exactly the same as Perelman's argument in Claim 4 of [2003, Section 2], where he proved the life of the standard solution is its caliber at initial time. See [Cao and Zhu 2006; Kleiner and Lott 2006; Morgan and Tian 2007] for details.

Our main theorem is this:

Theorem 2.4. There exists no noncollapsing, noncompact ancient solution with strictly positive curvature having a type I singularity. 
We argue by contradiction. Assume there is such a solution $X$. Since we assumed positive curvature, $X$ is diffeomorphic to $\mathbb{R}^{3}$. Its structure is described in Proposition 1.5; see Figure 1.

First, in the next two lemmas, under the assumption that $X$ as above exists, we construct an ancient solution with a global curvature bound similar to (0.2).

Lemma 2.5. We have

$$
\liminf _{t \rightarrow T^{-}}(T-t) \cdot \inf _{x} R(x, t)>0 .
$$

In particular, there exists $C, c>0$ so that for all $t$ close to $T$ and all $x$,

$$
c \leq(T-t) R(x, t) \leq C .
$$

Proof. By Lemma 2.3, at time $t$ the caliber of $(X, g(t))$ is $T-t$. Therefore

$$
\lim _{x \rightarrow \infty} R(x, t)=\frac{1}{T-t} .
$$

We claim there is a universal constant $c$ so that for all $y \in X$,

$$
R(y, t) \geq c \lim _{x \rightarrow \infty} R(x, t)=\frac{c}{T-t} .
$$

As in many occasions in Perelman's papers, for example, the last paragraph in 11.7 of [Perelman 2002], this follows from Yau's volume comparison argument with base point at infinity. For the reader's convenience, we give a sketch:

By rescaling, we can assume $T-t=1$. Take a ray $\gamma$ with $\gamma(0)=y$. If the distance $s_{1}$ is sufficiently large, a neighborhood of $\gamma\left(s_{1}\right)$ is close to a (piece of) cylinder with caliber $T-t$. Take $s_{2} \gg s_{1}$, let $p=\gamma\left(s_{2}\right)$, and define the one-direction annulus

$$
A\left(p, \delta_{1}, \delta_{2}\right)=\left\{x \in X \mid \delta_{1} \leq d(x, p) \leq \delta_{2}, \liminf _{s \rightarrow \infty} d(\gamma(s), x)-\left(s-s_{2}\right) \geq 0\right\}
$$

A volume comparison based at $p$ implies that

$$
\frac{\operatorname{Vol} A\left(p, s_{2}-D, s_{2}+D\right)}{\operatorname{Vol} A\left(p, s_{2}-s_{1}-D, s_{2}-s_{1}+D\right)} \leq \frac{\int_{s_{2}-D}^{s_{2}+D} r^{2} d r}{\int_{s_{2}-s_{1}-D}^{s_{2}-s_{1}+D} r^{2} d r} \approx 1,
$$

where $D \gg \sqrt{T-t}$ while $D \ll s_{1} \ll s_{2}$. Since $A\left(p, s_{2}-s_{1}-D, s_{2}-s_{1}+D\right)$, which contains $\gamma\left(s_{1}\right)$, is approximately a cylinder of length $2 D$ and caliber $T-t$, we see that

$$
\operatorname{Vol} A\left(p, s_{2}-s_{1}-D, s_{2}-s_{1}+D\right) \sim 2 D(T-t) \ll D^{3} .
$$

By Perelman's compactness theorem for ancient solutions (see the proof of 11.7 of [Perelman 2002]), there is a constant $N$ such that on any ancient solution,

$$
R(b) \leq N \quad \text { whenever } R(a)=1 \text { and } d(a, b) \leq 1 .
$$


Therefore if $R(y, t)$ is very small, say $R(y, t) \leq c \leq D^{-2} N^{-1}$, we have $R \leq D^{-2}$ on $B(y, t, D)$. So by the noncollapsing assumption we have

$$
\operatorname{Vol} A\left(p, s_{2}-D, s_{2}+D\right) \geq \operatorname{Vol} B(y, t, D) \geq \tau D^{3} .
$$

This, together with (2.12), contradicts (2.11).

Lemma 2.14. If $X$ is of type I, there exists a noncompact, noncollapsing ancient solution $X^{\prime}$ of positive curvature that goes singular at time 0 , and for all $x \in X^{\prime}$ and $t \leq 0$ we have

$$
c \leq|t| \cdot R(x, t) \leq C .
$$

Proof. Let $t_{i} \rightarrow T^{-}$. Pick a sequence of points $\left(x_{i}, t_{i}\right) \in X$ in the cap region, that is, at the $X_{C}$ region; see Proposition 1.5. Rescale $R\left(x_{i}, t_{i}\right)$ to 1 and get a pointed limit $X^{\prime}$. Observe that $X^{\prime}$ is noncompact, does not split, and has positive curvature. We then translate the singular time of $X^{\prime}$ to 0 , and the conclusion follows from the previous lemma.

We can then assume $X$ satisfies (2.15) and reduce Theorem 2.4 to the following:

Theorem 2.16. There is no noncollapsing, noncompact, ancient solution, with bounded positive curvature on each time interval $(-\infty, t]$ with $t<0$, satisfying the following:

(1) The solution goes singular at time $T=0$.

(2) For all $(x, t)$ we have $c \leq|t| \cdot R(x, t) \leq C$.

We break the proof into a sequence of lemmas. In view of Proposition 1.5, at time $t$ when $x \in X_{C}$, we say $x$ is on the cap at time $t$. When $x \in X_{T}$, we say $x$ is the center of a tube at time $t$; see Figure 1 . When the curvature is strictly positive, $X_{C}$ is diffeomorphic to the solid ball $D^{3}$. A point $x$ may be on the cap at one time and be the center of a tube at another time.

Remember Perelman's $l$ functional: fix a base point $(p, t)$, for $\left(q, t^{\prime}\right)$ with $t^{\prime}<t$, write $\tau=t-t^{\prime}$. Then define

$$
l(q, \tau)=\inf _{\gamma} \frac{1}{2 \sqrt{\tau}} \int_{0}^{\tau} \sqrt{s}\left(R(\gamma(s), s)+\left|\gamma^{\prime}(s)\right|^{2}\right) d s .
$$

The infimum is taken among all paths $\gamma:[0, \tau] \rightarrow M$ such that $\gamma(0)=p, \gamma(\tau)=q$.

Lemma 2.17. Let $\left(p_{i}, t_{i}\right)$ be a sequence of points with $t_{i} \rightarrow-\infty$ that are on the cap. Fix a time, say -1 . The points $\left(p_{i},-1\right)$ goes to space infinity when $i \rightarrow \infty$.

Proof. If this is not true, assume $\left(p_{i},-1\right) \rightarrow(p,-1)$. Take a fixed $\varepsilon>0$ and let $(p,-1+\varepsilon)$ be the base point. Take the constant curve $p_{i}$ from inverse time $\tau=\left(-1-t_{i}\right)+\varepsilon$ to $\varepsilon$, and connect with a path from $\left(p_{i},-1\right)$ to $(p,-1+\varepsilon)$. We 
get a piecewise smooth curve $\gamma_{i}$. Computing Perelman's $l$ integral on $\gamma_{i}$, we get, for all $\tau \gg 0$,

$$
l\left(p_{i}, \tau\right)=\frac{c(\varepsilon)}{2 \sqrt{\tau}}+\frac{1}{2 \sqrt{\tau}} \int_{\varepsilon}^{\tau} \sqrt{s} R d s \leq c^{\prime}(\varepsilon)+\frac{1}{2 \sqrt{\tau}} \int_{0}^{\tau} \sqrt{s} \frac{C}{s+c} d s \leq C .
$$

Here we used the assumption (2) in Theorem 2.16.

Thus by Perelman's asymptotic soliton theorem [2002, Proposition 11.2] (see also [Kleiner and Lott 2006; Morgan and Tian 2007; Chow et al. 2009]), if we take a rescaled limit at $\left(p_{i}, t_{i}\right)$, we get a nonflat shrinking soliton. The limit soliton cannot be compact, and since every $\left(p_{i}, t_{i}\right)$ is on the cap, the limit does not split, that is, the limit soliton is of positive curvature. By [Perelman 2003, Section 1], shrinking solitons are either space forms, or $S^{2} \times \mathbb{R}$, or $S^{2} \times \mathbb{R} / \mathbb{Z}_{2}$, none of which contains a cap diffeomorphic to the disc $D^{3}$. This is a contradiction.

Recall Perelman's reduced volume, defined by

$$
\widetilde{V}(\tau)=\tau^{-n / 2} \int_{M} e^{-l(x, \tau)} d x .
$$

Lemma 2.19. Given any $C>0$, there exists $\varepsilon$ with the following property. Let $\tau>2$ and let $(M, p)$ be an ancient solution with $\sup R \leq C$ at time -1 ; assume also that

$$
\widetilde{V}(\tau) \leq \widetilde{V}(2 \tau)+\varepsilon
$$

and $l(q, \tau) \leq C$, with the base point of $l$ and $\widetilde{V}$ taken at $(p,-1)$. Then, after rescaling by $R(q, \tau)$, a neighborhood of $(q, \tau)$ is close to a large subset in a shrinking soliton. Here $\tau=-1-t$ is the inverse time.

Proof. This follows exactly the proof of [Perelman 2002, Proposition 11.2], where, once there is a bound $l(q, \tau) \leq n / 2$, one can rescale down by $\tau$ and take the limit. (See [Ye 2008] for more details, especially about the bounds in $l$.) We can adapt this argument using the bound $l(q, \tau) \leq C$.

Perelman then uses the fact that $\widetilde{V}$ is decreasing in $\tau$, so when taking the scaledown limit, $\widetilde{V}$ converges to a constant function (independent of time). Then the differential inequalities (7.13)-(7.14) in [Perelman 2002] become equalities, thus the limit is a shrinking soliton. In our situation, we make a similar argument by contradiction. Take $\varepsilon_{i} \rightarrow 0$ and a sequence of ancient solutions $M_{i}$ and times $\tau_{i}$ such that (2.20) holds for $\tau=\tau_{i}$. After passing to a subsequence, we get a limit space, and $\widetilde{V}$ converges to a constant since $\varepsilon_{i} \rightarrow 0$. Then we use [Perelman 2002, (7.13), (7.14)] to conclude that the limit is a shrinking soliton and get a contradiction.

Lemma 2.21. Assume there is an ancient solution $X$ as in Theorem 2.16. For any $\varepsilon>0$, there exists $N>0$ such that for any point $(p,-1)$ that is sufficiently far into 
space infinity, we have

$$
\frac{2}{e}(4 \pi)^{3 / 2}<\widetilde{V}(\tau) \leq \widetilde{V}(2 \tau)+\frac{\varepsilon}{2}<\frac{2}{e}(4 \pi)^{3 / 2}+\varepsilon,
$$

for all $\tau>N$. Here we take $(p,-1)$ as the base point for computing $l$ and $\widetilde{V}$.

Proof. At time $t$, at space infinity the solution looks like a tube of caliber $|t|$. No matter where the base point $(p, t) \in X$ for $l$ is located, we claim that

$$
\widetilde{V}_{X}(\infty)=\lim _{\tau \rightarrow \infty} \widetilde{V}_{X}(\tau)=\frac{2}{e}(4 \pi)^{3 / 2} .
$$

In fact, pick any base point $(p, t)$, translate the time $t$ to 0 ; then we have the inverse time $\tau$. Applying [Perelman 2002, Proposition 11.2], we scale down this solution and take a limit; the limit $Z$ is a shrinking soliton, called the asymptotic soliton. $Z$ is noncompact, so it must be $Z=S^{2} \times \mathbb{R}\left(S^{2} \times \mathbb{R} / \mathbb{Z}_{2}\right.$ does not happen because the curvature is positive). Therefore $Z$ is an evolving cylinder that goes singular at rescaled inverse time $\tilde{\tau}=0$. Let $\widetilde{V}_{\text {cylinder }}$ be the limit of $\widetilde{V}_{X}$ on $Z$. By [Perelman 2002], the limit

$$
\widetilde{V}_{\text {cylinder }}(\tilde{\tau})=\widetilde{V}_{\text {cylinder }}(\infty)
$$

does not depend on $\tilde{\tau}$. The precise value of $\widetilde{V}_{\text {cylinder }}(\tilde{\tau})$ is easy to compute; see for example [Cao et al. 2004; Kleiner and Lott 2006; Morgan and Tian 2007]. Briefly, notice that Ric $+\operatorname{Hess}_{l}=g /(2 \tilde{\tau})$ on the cylinder, and pick $\tilde{\tau}=1$. At this time we have $R=1$. First taking the trace along the $S^{2}$ factor, we see that $l$ is constant on each $S^{2}$ slice. Then taking the trace on $Z$, we see that $l$ is a quadratic polynomial along the $\mathbb{R}$ direction. Choosing a suitable origin and noticing that the differential inequality (7.14) of [Perelman 2002] becomes an equality, we can completely determine $l$ :

$$
l_{\text {cylinder }}=1+x^{2} / 4,
$$

where $x$ is the coordinate in the $\mathbb{R}$ direction. Integrating we have

$$
\begin{aligned}
\widetilde{V}_{\text {cylinder }}(\infty) & =\widetilde{V}_{\text {cylinder }}(1) \\
& =\int_{\text {cylinder }} e^{-l}=\operatorname{Vol} S^{2} \cdot \int_{-\infty}^{\infty} e^{-1-x^{2} / 4} d x=\frac{2}{e}(4 \pi)^{3 / 2} .
\end{aligned}
$$

(Here Vol $S^{2}=8 \pi$ since $R=1$ ). By Perelman's growth estimate for $l$ [Ye 2008, Lemma 3.2], one can bound the contribution to $\widetilde{V}$ outside any given distance. Moreover the scale-down limit of $l$ is just $l_{\text {cylinder }}$. Hence

$$
\widetilde{V}_{X}(\infty)=\widetilde{V}_{\text {cylinder }}(\infty)=2 e^{-1}(4 \pi)^{3 / 2} .
$$

Picking any $(z,-1)$ in an evolving cylinder $Z$ as the base point for computing $l$, we get the same limit value $\widetilde{V}_{Z}(\infty)=2 e^{-1}(4 \pi)^{3 / 2}$. Although both $\widetilde{V}_{Z}$ and $\widetilde{V}_{\text {cylinder }}$ 
are computed on the evolving cylinder, they are different in that $\widetilde{V}_{\text {cylinder }}$ is defined as the limit of reduced volumes over rescaled manifolds, which essentially used the singularity as base point; $\widetilde{V}_{Z}$ uses an ordinary point $(z,-1)$ as the base point. Therefore $\widetilde{V}_{\text {cylinder }}(\tau)$ does not depend on $\tau$, while $\widetilde{V}_{Z}(\tau)$ does.

There exists $N$ such that

$$
\widetilde{V}_{Z}(N) \leq \frac{2}{e}(4 \pi)^{3 / 2}+\frac{\varepsilon}{8}
$$

Moreover, there is a radius $D \gg \sqrt{N}$ such that, on $Z$,

$$
\widetilde{V}_{Z}(N)-\frac{\varepsilon}{8} \leq \int_{B(z,-1-N, D)} N^{-n / 2} e^{-l} \leq \widetilde{V}_{Z}(N) \leq \frac{2}{e}(4 \pi)^{3 / 2}+\frac{\varepsilon}{8} .
$$

Observe that on cylinders, all $l$-geodesics connecting the base point $(z,-1)$ to points in $B(z,-1-N, 2 D)$ stay entirely in $B(z,-1-N, 2 D)$.

If we take pointed limit with base points $\left(p_{i},-1\right) \in X$ going to space infinity, we get an approximate cylinder of caliber 1 . Therefore if we go sufficiently far into the space infinity, we see the ball $B$ centered at $(p,-1)$ with radius $2 D$ satisfies that $B \times[-1-N,-1]$ is close to the corresponding part in space-time of the evolving cylinder $Z$. By the Schwartz inequality, we see that all $l$ geodesics to points in $B(p,-1-N, D)$ stay entirely in $B(p,-1-N, 3 D / 2)$ for sufficiently large $i$.

By Perelman's growth estimate on $l$ [2008, Lemma 3.2], the contribution to $\widetilde{V}$ of points outside $B \times[-1-N,-1]$ can be nicely controlled:

$$
\widetilde{V}_{X}(N)-\frac{\varepsilon}{8} \leq \int_{B(p,-N-1, D)} N^{-n / 2} e^{-l_{X}}<\widetilde{V}_{X}(N) .
$$

But recall from [Perelman 2002, (7.12), (7.13)] that $\widetilde{V}$ is a decreasing function in $\tau$, so $\widetilde{V}_{X}(N) \geq \widetilde{V}_{X}(\infty)$. Since the neighborhood $B \times[-1-N,-1]$ is $C^{6}$-close to an evolving cylinder, we have

$$
\left|\int_{B(p,-N-1, D)} N^{-n / 2} e^{-l_{X}}-\int_{B(z,-N-1, D)} N^{-n / 2} e^{-l_{Z}}\right| \leq \frac{\varepsilon}{8} .
$$

Both these above integrals are close to $\widetilde{V}_{X}(\infty)$. Combining (2.23), (2.27) and (2.28) with the fact that $\widetilde{V}$ is a decreasing function, we get the conclusion (2.22) for all $\tau>N$.

Proof of Theorem 2.16. Take a sequence of points $\left(p_{i}, t_{i}\right)$ on the cap, with $t_{i} \rightarrow$ $-\infty$. By Lemma 2.17, for sufficiently large $i$, and $\left|t_{i}\right|>N+1$, where $N$ is the number in Lemma 2.21, we see that $\left(p_{i},-1\right)$ is so far into space infinity that Lemma 2.21 applies. Using $\left(p_{i},-1\right)$ as the base point for $l$, we conclude that

$$
\widetilde{V}(\tau) \leq \widetilde{V}(2 \tau)+\varepsilon
$$


for all $\tau>N$. In particular we take $\left(p_{i},-1\right)$ as the base point and use $\tau=-1-t_{i}$. The type I assumption implies a bound of $l$ at $\left(p_{i}, t_{i}\right)$, evaluated on the constant curve $p_{i}$; see the computation (2.18). Therefore by Lemma 2.19, after rescaling by $R\left(p_{i}, t_{i}\right)$ we see that a big neighborhood of $\left(p_{i}, t_{i}\right)$ is close to a set in a shrinking soliton - either a space form or a cylinder (or its $\mathbb{Z}_{2}$ quotient). But this is impossible because $\left(x_{i}, t_{i}\right)$ is in a cap region $X_{C}$ that is diffeomorphic to the unit ball $D^{3}$.

\section{Forward limit and eternal solutions}

To get more concrete information, especially for taking forward limits, we need the following lemma, which could be used as an alternative definition of a degenerate singularity. This is very similar to [2003, Lemma 4.3]; see also [Cao and Zhu 2006; Kleiner and Lott 2006; Morgan and Tian 2007].

Lemma 3.1. Assume a degenerate singularity happens at time $T$ and the solution does not become extinct everywhere at $T$. There exists a positive real number $\varepsilon>0$ (depending on the solution) and a compact set $B \subset M$ diffeomorphic to a solid 3ball, such that some neighborhood of $\partial B$ is $10^{-2}$-close to a cylinder of scalar curvature 1 and length 100, after rescaling the metric by $R(p, t)$ for any $p \in \partial B$ and for all $t \in[T-\varepsilon, T)$. We also have

$$
\limsup _{t \rightarrow T^{-}} \sup _{p \in \partial B} R(p, t)<\infty .
$$

Moreover, let $p(t) \in B$ be a point such that

$$
d_{g(t)}(p(t), \partial B)=\sup _{p \in B} d_{g(t)}(p, \partial B) .
$$

Then

$$
\lim _{t \rightarrow T^{-}} R(p(t), t)=\infty .
$$

Proof. Let $\left(x_{i}, t_{i}\right)$ be a sequence of points as in Definition 1.7. Since $M$ is compact and the metric expands in a controlled way, we can assume $x_{i} \rightarrow x_{\infty}$. Then

$$
\lim _{t \rightarrow T^{-}} R\left(x_{\infty}, t\right)=\infty .
$$

In fact, if this is not true, then for some $C>0$, there exists $t$ such that $T-t$ is arbitrarily small while $R\left(x_{\infty}, t\right)<C$; we pick such a time $t^{*}$ that is sufficiently close to $T$. By the gradient estimate (1.4) in the space direction, some neighborhood $\mathcal{N}$ of $\left(x_{\infty}, t^{*}\right)$ has bounded curvature. Because we have chosen $t^{*}$ sufficiently close to $T$, by the time-direction gradient estimate in (1.4), $\mathcal{N}$ has a uniform curvature bound on the time interval $\left(t^{*}, T\right)$. So on $\mathcal{N}$, the metrics from time $t^{*}$ to $T$ are 
uniformly equivalent. In particular, for sufficiently large $i$ we have $x_{i} \in \mathcal{N}$; this contradicts to the fact $R\left(x_{i}, t_{i}\right) \rightarrow \infty$.

It was assumed that the solution does not become extinct everywhere at time $T$. As we have seen above, by the gradient estimate (1.4), near time $T$, the curvature cannot increase or decrease suddenly. So there is a point $y$ such that

$$
\limsup _{t \rightarrow T^{-}} R(y, t)=C<\infty .
$$

Pick a time $t^{*}$ that is sufficiently close to $T$ and such that

$$
R\left(x_{\infty}, t^{*}\right)>\eta^{-8} \cdot \max \left\{r_{0}^{-2}, C\right\}
$$

here $r_{0}$ is the canonical neighborhood parameter in Proposition 1.3 and $\eta$ is the (small) constant in Perelman's splitting argument (Proposition 1.8). Take a minimal $g\left(t^{*}\right)$-geodesic $\gamma$ from $y$ to $x_{\infty}$, parametrized by $s$. Choose $r_{1}$ so that $r_{1}^{-2}=\eta^{-2}$. $\max \left\{r_{0}^{-2}, C\right\}$, and let

$$
s_{0}=\sup \left\{s \mid R\left(\gamma(s), t^{*}\right)=r_{1}^{-2}\right\}, \quad s_{1}=\sup \left\{s \mid R\left(\gamma(s), t^{*}\right)=\eta^{-6} r_{1}^{-2}\right\} .
$$

Since $t^{*}$ is sufficiently close to $T$, by applying the gradient estimate (1.4), we can assume that

$$
R\left(\gamma\left(s_{0}\right), t\right) \leq 2 r_{1}^{-2}
$$

on the time interval $\left(t^{*}, T\right)$. By Proposition 1.8, the canonical neighborhood of $\gamma\left(s_{0}\right)$ at time $t^{*}$ is a tube, with radius $r_{1} \sqrt{2}$. Now remove a center sphere $S^{2}$ in this tube at $\gamma\left(s_{0}\right)$ from the manifold $\left(M, g\left(t^{*}\right)\right)$ to get an incomplete Riemannian manifold, possibly with two components. Pick the component that contains $x_{\infty}$ and take its closure $M^{*}$. So

$$
\gamma\left(\left[s_{0}, d\left(y, x_{\infty}\right)\right]\right) \subset M^{*} .
$$

The boundary $\partial M^{*}$ is either connected or has two components, each diffeomorphic to $S^{2}$. Write $S=\partial M^{*}$ when $\partial M^{*}$ is connected; when $\partial M^{*}$ has two components, let $S$ be the component of $\partial M^{*}$ closer to $\gamma\left(s_{0}+\delta\right)$, where $\delta=R\left(\gamma\left(s_{0}\right), t^{*}\right)^{-1 / 2}$. In other words, $S$ is the departure point of the part of $\gamma$ that runs towards $x_{\infty}$.

The Ricci flow still runs on this manifold (it runs on the original manifold $M$, and we just removed a sphere). For all $t \in\left[t^{*}, T\right)$, pick a point $q(t)$ furthest from $S$ :

$$
d_{t}(q(t), S)=\max \left\{d(x, S) \mid x \in M^{*}\right\} .
$$

Let $\alpha$ be a minimum $g(t)$-geodesic from $S$ to $q(t)$. As we will see, $\alpha$ is close to $\gamma$.

At time $t^{*}, S$ is the start of a piece of tube, and $\gamma$ passes through $S$. From $S$ to $\gamma\left(s_{1}\right)$, which is $\left|s_{1}-s_{0}\right|$ away from $S$, the scalar curvature changes from $r_{1}^{-2}$ to $\eta^{-2} r_{1}^{-2}$. By Proposition 1.8, this part of $\gamma$ is close to $\gamma$. There are two cases: 


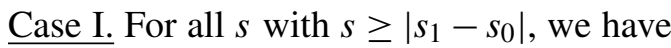

$$
R\left(\alpha(s), t^{*}\right)>\frac{1}{2} \eta^{-2} r_{1}^{-2} .
$$

In this case, if $\partial M^{*}$ has another component $S^{\prime}$, the canonical neighborhoods of $\alpha(s)$ do not intersect $S^{\prime}$. In fact a neighborhood of $S^{\prime}$ is very close to a long tube with scalar curvature $r_{1}^{-2}$ - the curvature (3.11) is too big: remember if we glue back the broken neck, $S^{\prime}$ will be identified with $S$.

On the other hand, (3.11) implies that every point of $\alpha$ has a canonical neighborhood. By the classification of canonical neighborhoods and Proposition 1.8, away from $q\left(t^{*}\right)$ (the end of $\alpha$ ), $\alpha$ is covered by tubes; near $q\left(t^{*}\right)$, the canonical neighborhoods of $\alpha(s)$ are diffeomorphic to a cap or to $R P^{3}-D^{3}$. Therefore $M^{*}$ is diffeomorphic to a solid ball $D^{3}$ or $R P^{3}-D^{3}$; in particular, $\partial M^{*}=S$ is connected.

Now $x_{\infty} \in M^{*}$, and the curvature at $\partial M^{*}=S$ remains bounded when $t \rightarrow T$ because $t^{*}$ is very close to $T$, we conclude that $x_{i} \in M^{*}$ for $i$ sufficiently large. So the possibility $M^{*}=R P^{3}-D^{3}$ is ruled out, because $M^{*}$ contains a cap diffeomorphic to $D^{3}$ and this cap contains the point $q\left(t^{*}\right)$ maximizing the distance function $d_{M^{*}}(\cdot, S)$. Therefore, we can just take $p(t)=q(t)$ and $B=M^{*}$.

Case II. There is a minimal $s^{\prime}$ such that $s^{\prime}>\left|s_{0}-s_{1}\right|$ and

$$
R\left(\alpha\left(s^{\prime}\right), t^{*}\right)=\frac{1}{2} \eta^{-2} r_{1}^{-2} \text {. }
$$

We will prove that this is impossible. Roughly speaking, $x_{\infty}$ is trapped between $\gamma\left(s_{1}\right)$ and $\alpha\left(s^{\prime}\right)$, so by Perelman's splitting argument (Proposition 1.8), the neighborhood near $x_{\infty}$ will not be "cap-like":

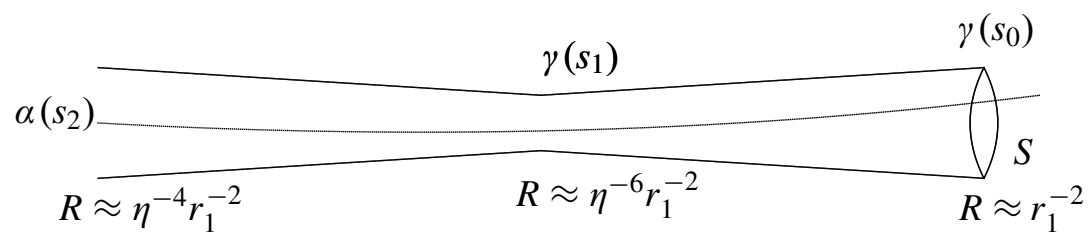

More precisly, choose a minimal $s_{2}$ such that $s_{2}>\left|s_{0}-s_{1}\right|$ and

$$
R\left(\alpha\left(s_{2}\right), t^{*}\right)=\eta^{-4} r_{1}^{-2} .
$$

Since we chose $t^{*}$ very close to $T$, by the gradient estimate (1.4), a neighborhood $\mathcal{N}_{2}$ of $\alpha\left(s_{2}\right)$ has a curvature bound

$$
R \leq 10 \eta^{-4} r_{1}^{-2}
$$

for all time $t \in\left[t^{*}, T\right)$. Again by Proposition 1.8, the canonical neighborhood $\mathcal{N}_{2}$ is a cylinder. Now cut $M^{*}$ at a center sphere at $\alpha\left(s_{2}\right)$ and take the component $\mathscr{C}$ that contains $\gamma\left(s_{1}\right)$. This $\mathscr{C}$ is just a piece of a cylinder; see again Proposition 1.8. 
In particular, by the choice of $\gamma\left(s_{1}\right)$, we have $x_{\infty} \in \mathscr{C}$. By (3.14), have uniform curvature bounds on the two ends of this cylinder on the time interval $\left[t^{*}, T\right)$. So for sufficiently large $i$, we have $x_{i} \in \mathscr{C}$.

On the other hand, also by (1.4), there is actually a lower bound

$$
\frac{1}{10} r_{1}^{-2} \leq R(x, t)
$$

for all $x \in \mathscr{C}$ and $t \in\left[t^{*}, T\right)$. This means for all time $t \in\left[t^{*}, T\right)$, any $x \in \mathscr{C}$ has a canonical neighborhood.

We already know that near the two ends, the canonical neighborhoods are both cylinders. Let $\alpha_{t}$ be a minimal $g(t)$-geodesic that connects the two ends of $\mathscr{C}$. For all $x \in \alpha_{t}$, the canonical neighborhood is a cylinder because a cap cannot contain a geodesic segment extending very far in both direction. Thus the union of all these cylinder neighborhoods is exactly $\mathscr{C}$. This contradicts the fact that the canonical neighborhood of $\left(x_{i}, t_{i}\right)$, which lies in $\mathscr{C}$, is a cap.

Assume a degenerate singularity happens at time $T$. By taking a rescaling limit at the point $(p(t), t)$ in Lemma 3.1, with $t \rightarrow T^{-}$, we get an ancient solution $X$. Since the canonical neighborhoods of $p(t)$ are of case (iv) in Proposition 1.3, the limit $X$ is necessarily of strictly positive curvature, otherwise the solution is $S^{2} \times \mathbb{R}$ or its $\mathbb{Z}_{2}$ quotient, by the strong maximum principle. By a translation in time, $X$ exists on $(-\infty, 0]$.

Let $T_{1}>0$ be the maximal time such that there is an extension of $X$ up to time $T_{1}$ and for all $t<T_{1}$, the curvature of $g(t)$ is positive and bounded. (Such an extension need not be unique.) Then $T_{1}$ is the caliber of $(X, g(0))$; see Lemma 2.3. A priori, when $0<T^{\prime}<T_{1}$ we do not know if the solution $(X, g(t))$, with $-\infty<t \leq T^{\prime}$, is a rescaling limit of the original Ricci flow on $M$.

Lemma 3.16. There exists an extension $(X, g(t))$ with $-\infty<t<T_{1}$, where $T_{1}$ is the caliber of $(X, g(0))$, such that for all $T^{\prime}>0$ with $T^{\prime}<T_{1}$, the ancient solution $X$ over time $\left(-\infty, T^{\prime}\right]$ is a rescaling limit of the original solution on $M$.

Proof. Let $T_{0}$ be the supremum of the set of times $T$ such that there is an extension of $X$ to time $T$ and the ancient solution $X$ over time $(-\infty, T)$ is a rescaling limit of the original solution on $M$.

We have $T_{0}>0$. In fact, a rescaling of $M$ is close to $(X, g(0))$ in the pointed $C^{3}$ topology. We can assume the rescaling is taken at $p(t)$ in Lemma 3.1, (remember that the original solution does not go singular unless $R(p(t), t)$ goes to infinity). Now the corresponding neighborhood $\mathcal{N}(t)$ of $p(t)$ contains a cap part $\mathcal{N}_{C}$ and a tube part $\mathcal{N}_{T}$, corresponding to the $X_{C}, X_{T}$ decomposition of $X$.

From the definition of $p(t)$ in (3.3), as long as the Ricci flow is running (at least for a short time when the metric geometry of $\mathcal{N}$ does not change too much), the global maximum point $p(t+\Delta t)$ of the distance function must remain in $\mathcal{N}$. 
The gradient estimate (1.4) implies that the curvature on $\mathcal{N}$ cannot go to infinity immediately, so $R(p(t+\Delta t), t+\Delta t)$ does not go to infinity immediately. During this time period the solution cannot go singular. Therefore we can take a definite short-time forward limit at $p(t)$. This limit extends $X$, so $T_{0}>0$.

We can repeat this argument (that is, view $T_{0}$ as time 0 ) as long as $X$ does not go singular; therefore we must have $T_{0}=T_{1}$, the caliber of $(X, g(0))$.

We would like to have $T_{1}=\infty$, so that $X$ is an eternal solution by Lemma 2.3. Assume this is not the case. Then the solution must develop a singularity at time $T_{1}$. By Theorem 2.4, this singularity is of type II as defined in [Hamilton 1995], that is,

$$
\limsup _{t \rightarrow T^{-}}\left(T_{1}-t\right) \cdot \sup _{x} R(x, t)=\infty .
$$

In all cases, by taking a further limit we will get an eternal solution in which the scalar curvature reaches maximum. This point-picking procedure is well known [Perelman 2002; 2003; Kleiner and Lott 2006; Chow et al. 2006]. We give a quick account here.

We will find a sequence of points $\left(p_{n}, t_{n}\right)$ such that when we rescale the solution by $R\left(x_{n}, t_{n}\right)$ (that is, first make $\left.R\left(x_{n}, t_{n}\right)=1\right)$, and then translate the time $t_{n}$ into 0 (that is, our base point is now $\left(p_{n}, 0\right)$ with $\left.R\left(p_{n}, 0\right)=1\right)$, then the solution exists on the interval $(-\infty, n]$ and

$$
1=R\left(p_{n}, 0\right) \geq\left(1-\frac{1}{n+1}\right) \sup \{R(x, t) \mid x \in X, t \leq n\} .
$$

In fact, start with $(p, 0)=\left(p^{(0)}, t^{(0)}\right)$ so that $R(p, 0)$ is maximal at time 0 , and

$$
\left(T_{1}-t^{(0)}\right) R\left(p^{(0)}, t^{(0)}\right)>(n+1)^{2}
$$

if $X$ is not eternal. Assume there is a point $p^{(1)}$ and a time $t^{(1)}$ such that

$$
R\left(p^{(1)}, t^{(1)}\right)>\left(1+\frac{1}{n}\right) R\left(p^{(0)}, t^{(0)}\right), \quad t^{(1)}-t^{(0)} \leq n R\left(p^{(0)}, t^{(0)}\right)^{-1} .
$$

Without loss of generality we assume that $R\left(p^{(1)}, t^{(1)}\right)$ is almost maximal at the time slice $t=t^{(1)}$. If $\left(p^{(1)}, t^{(1)}\right)$ does not satisfy our requirement, we continue to find $\left(p^{(2)}, t^{(2)}\right), \ldots$ In general,

$$
\begin{gathered}
R\left(p^{(k)}, t^{(k)}\right)>\left(1+\frac{1}{n}\right)^{k} R\left(p^{(0)}, t^{(0)}\right), \\
t^{(k)}-t^{(0)} \leq n \sum_{i=0}^{k-1}\left(1+\frac{1}{n}\right)^{-i} R\left(p^{(0)}, t^{(0)}\right)^{-1}<n(n+1) R\left(p^{(0)}, t^{(0)}\right)^{-1}
\end{gathered}
$$

This procedure must stop at some finite $k$, since the solution $X$ exists up to time $(n+1)^{2} R\left(p^{(0)}, t^{(0)}\right)^{-1}$. Hence there is a curvature bound at the time slice $t=$ 
$n(n+1) R\left(p^{(0)}, t^{(0)}\right)^{-1}$ (see also Theorem 11.4 in [Perelman 2002]); by Hamilton's Harnack inequality [Hamilton 1993b], this scalar curvature bound actually holds for all $t \leq n(n+1) R\left(p^{(0)}, t^{(0)}\right)^{-1}$.

Taking a pointed limit $Z$ of $\left(X,\left(p_{n}, t_{n}\right), R\left(p_{n}, t_{n}\right) g\right)$, we get an eternal solution on which the maximal of scalar curvature is reached. Therefore we can apply Hamilton's theorem on eternal solutions [1993a] to conclude that the limit $Z$ is a steady gradient soliton. By Lemma 3.16 we finally have:

Theorem 3.23. Assume $g(t)$ develops a degenerate singularity at time $T$. Then a rescaling limit of $g(t)$ towards time $T$ is a steady gradient soliton.

Proof. We have already proved this when the solution does not become extinct everywhere at time $T$.

The other case, that $M$ becomes extinct everywhere at time $T$, is actually easier. In fact the solution does not become extinct unless the everywhere the curvature goes to infinity; so there is no difficulty in obtaining a forward time limit - one can take the limit as long as the local curvature remain finite, and in this case no singularities occur at far away.

On the other hand, when we take the limit as before and get an ancient solution $X$ with nonnegative curvature, $X$ is noncompact. For otherwise, $X$ would be diffeomorphic to $S^{3}$ or $P R^{3}$. By taking a forward limit we see that the original solution must be of strictly positive curvature near time $T$; in particular it will get rounder and rounder and cannot be a degenerated singularity. So the proof goes like the first case.

Corollary 3.24. Our Definition 1.7 of a degenerate singularity is equivalent to the definition of a type II singularity.

Perelman [2003, Section 2] constructed a "standard solution". It is not an ancient solution.

Corollary 3.25. The rescaling limit of Perelman's standard solution [2003] is the Bryant soliton.

Proof. Perelman's standard solution $X$ goes singular at time 1. This singularity must be of type II, otherwise by taking a rescaled limit we get an ancient solution satisfying the conditions in Theorem 2.16, and such solutions do not exist.

It is known that Perelman's standard solution $X$ is unique; see [Perelman 2003; Morgan and Tian 2007, Chapter 12]. Hence $X$ is radially symmetric, in particular, $X$ is the union of $\mathrm{SO}(3)$ orbits. One can write the metric of $X$ as

$$
d r^{2}+f(r)^{2} d S^{2}
$$

where $f$ is a warping function such that $f(0)=0, f^{\prime}(r)>0$, and $f^{\prime \prime}(r)<0$ for $r>0$. 
Now as in Theorem 3.23, there is a sequence $x_{i} \in X$ such that after rescaling by $R\left(x_{i}, t_{i}\right)$, we can take a pointed limit for $\left(X, x_{i}, t_{i}\right)$ and get a steady gradient soliton $\left(Z, z_{0}\right)$ of positive curvature such that $R\left(z_{0}\right)=1$. It is clear that $Z$ is not compact.

After rescaling $X$ by $R\left(x_{i}, t_{i}\right)$, the radius of the $\mathrm{SO}(3)$ orbit passing through $\left(x_{i}, t_{i}\right)$ remains uniformly bounded. Otherwise (taking into account that $R$ is constant on any $\mathrm{SO}(3)$ orbit), in the limit we would have for $Z$

$$
\limsup _{z \rightarrow \infty} R(z) \geq R\left(z_{0}\right)=1,
$$

which is not possible when $Z$ is a steady soliton of positive curvature - see Lemma 2.3 and also [Hamilton 1995; Perelman 2002].

Let $x^{*}$ be the (time-independent) center of $X$. We claim that

$$
R\left(x_{i}, t_{i}\right) d\left(x^{*}, x_{i}, t_{i}\right)^{2}
$$

remains bounded. Assume this is not true. Perelman's compactness theorem [2002, 11.7] implies that there is a uniform curvature growth estimate for all ancient solutions of positive curvature. In particular,

$$
R\left(z_{0}\right) \Psi\left(R\left(z_{0}\right) d\left(z, z_{0}\right)^{2}\right) \leq R(z) \leq R\left(z_{0}\right) \Phi\left(R\left(z_{0}\right) d\left(z, z_{0}\right)^{2}\right),
$$

where $\Psi, \Phi$ are universal positive functions. Since the warping function $f$ is monotonic and concave, by computing the curvature of $X$ in term of $f$ we see that, within finite distance, there are uniform upper and lower bounds for the diameters of the $\mathrm{SO}(3)$ orbits. In particular, $Z$ is diffeomorphic to $S^{2} \times \mathbb{R}$, but this is impossible since $Z$ is of positive curvature.

Therefore we can take the rescaled limit using the base point $\left(x^{*}, t_{i}\right)$ instead. In particular, the steady soliton $Z$ is radially symmetric. By the discussion in [Chow et al. 2006], $Z$ must be the Bryant soliton.

Finally we mention that a result analogous to Lemma 3.1 for $R P^{3}-D^{3}$ caps also holds. It is easy to prove that locally the singularity is of type I and a rescaling limit is $S^{2} \times \mathbb{R} / \mathbb{Z}_{2}$. One can get an example of such a singularity by taking $\mathbb{Z}_{2}$ quotients of the examples of nondegenerate singularities in [Chow and Knopf 2004, Chapter 2]; this is also a singularity like a horn growing out of $S^{3}$, but the tip of the horn is actually $R P^{3}-B^{3}$. We do not call this case degenerate.

\section{Acknowledgments}

After finishing this note, it came to my attention that during his spring 2003 seminars at Stony Brook, Perelman stated a result on ancient solutions that is similar to Theorem 2.4 in this paper. As far as I know, he did not circulate its proof; I am very grateful to Professor Ni for informing me of this. 
We thank Professors B. Chow, D. Knopf, L. Ni, G. Wei and R. Ye for helpful conversations.

\section{References}

[Angenent and Knopf 2004] S. Angenent and D. Knopf, "An example of neckpinching for Ricci flow on $S^{n+1}$, Math. Res. Lett. 11:4 (2004), 493-518. MR 2005e:53099 Zbl 1064.58022

[Angenent and Knopf 2007] S. B. Angenent and D. Knopf, "Precise asymptotics of the Ricci flow neckpinch", Comm. Anal. Geom. 15:4 (2007), 773-844. MR 2395258 Zbl 1145.53049

[Cao and Zhu 2006] H.-D. Cao and X.-P. Zhu, "A complete proof of the Poincaré and geometrization conjectures-application of the Hamilton-Perelman theory of the Ricci flow", Asian J. Math. 10:2 (2006), 165-492. MR 2008d:53090 Zbl 1146.93371

[Cao et al. 2004] H. Cao, R. Hamilton, and T. Ilmanen, "Gaussian densities and stability for some Ricci solitons", preprint, 2004. arXiv math.DG/0404165

[Chow and Knopf 2004] B. Chow and D. Knopf, The Ricci flow: an introduction, Math. Surveys and Monogr. 110, Amer. Math. Soc., Providence, RI, 2004. MR 2005e:53101 Zbl 1086.53085

[Chow et al. 2006] B. Chow, P. Lu, and L. Ni, Hamilton's Ricci flow, Graduate Studies in Math. 77, Amer. Math. Soc., Providence, RI, 2006. MR 2008a:53068 Zbl 1118.53001

[Chow et al. 2007] B. Chow, S.-C. Chu, D. Glickenstein, C. Guenther, J. Isenberg, T. Ivey, D. Knopf, P. Lu, F. Luo, and L. Ni, The Ricci flow: techniques and applications, I: Geometric aspects, Math. Surveys and Monogr. 135, Amer. Math. Soc., Providence, RI, 2007. MR 2008f:53088 Zbl 05149301

[Chow et al. 2009] B. Chow, S.-C. Chu, D. Glickenstein, C. Guenther, J. Isenberg, T. Ivey, D. Knopf, P. Lu, F. Luo, and L. Ni, The Ricci flow: techniques and applications, III: Geometric-analytic aspects, Math. Surveys and Monogr., Amer. Math. Soc., Providence, RI, 2009.

[Garfinkle and Isenberg 2008] D. Garfinkle and J. Isenberg, "The modeling of degenerate neck pinch singularities in Ricci flow by Bryant solitons", J. Math. Phys. 49:7 (2008), 073505, 10. MR 2432043

[Gu and Zhu 2007] H. Gu and X. Zhu, "The existence of type II singularities for the Ricci flow on $S^{n+1}$, preprint, 2007. arXiv 0707.0033v1

[Hamilton 1982] R. S. Hamilton, "Three-manifolds with positive Ricci curvature", J. Differential Geom. 17:2 (1982), 255-306. MR 84a:53050 Zbl 0504.53034

[Hamilton 1993a] R. S. Hamilton, "Eternal solutions to the Ricci flow", J. Differential Geom. 38:1 (1993), 1-11. MR 94g:58043 Zbl 0792.53041

[Hamilton 1993b] R. S. Hamilton, "The Harnack estimate for the Ricci flow", J. Differential Geom. 37:1 (1993), 225-243. MR 93k:58052 Zbl 0804.53023

[Hamilton 1995] R. S. Hamilton, "The formation of singularities in the Ricci flow", pp. 7-136 in Surveys in differential geometry (Cambridge, MA, 1993), vol. II, edited by C.-C. Hsiung and S.-T. Yau, Int. Press, Cambridge, MA, 1995. MR 97e:53075 Zbl 0867.53030

[Hamilton 1999] R. S. Hamilton, "Nonsingular solutions of the Ricci flow on three-manifolds", Comm. Anal. Geom. 7:4 (1999), 695-729. MR 2000g:53034 Zbl 0939.53024

[Kleiner and Lott 2006] B. Kleiner and J. Lott, “Notes on Perelman's papers”, preprint, 2006. arXiv math.DG/0605667

[Morgan and Tian 2007] J. Morgan and G. Tian, Ricci flow and the Poincaré conjecture, Clay Math. Monogr. 3, Amer. Math. Soc., Providence, RI, 2007. MR 2008d:57020 Zbl 05188193 
[Perelman 2002] G. Perelman, "The entropy formula for the Ricci flow and its geometric applications”, preprint, 2002. arXiv math.DG/0211159

[Perelman 2003] G. Perelman, "Ricci flow with surgery on three-manifolds", preprint, 2003. arXiv math.DG/0303109

[Shi 1989a] W.-X. Shi, "Deforming the metric on complete Riemannian manifolds", J. Differential Geom. 30:1 (1989), 223-301. MR 90i:58202 Zbl 0676.53044

[Shi 1989b] W.-X. Shi, "Ricci deformation of the metric on complete noncompact Riemannian manifolds”, J. Differential Geom. 30:2 (1989), 303-394. MR 90f:53080 Zbl 0686.53037

[Ye 2008] R. Ye, "On the $l$ function and the reduced volume of Perelman, I", preprint, 2008, Available at http://www.math.ucsb.edu/ yer.

Received May 7, 2008. Revised December 9, 2008.

\author{
YU DING \\ DePartment of MATHEMATiCS AND Statistics \\ CALIFORNIA State UNIVERSITY \\ LONG BEACH, CA 90840 \\ UNITEd STATES \\ yding@csulb.edu \\ http://www.csulb.edu/ yding
}

